\title{
DRUG-seq Provides Unbiased Biological Activity Readouts for Drug Discovery
}

Jingyao $\mathrm{Li}^{1^{*}}$, Daniel J. Ho ${ }^{1^{*}}$, Martin Henault ${ }^{2}$, Chian Yang ${ }^{2}$, Marilisa Neri' ${ }^{2}$, Robin $\mathrm{Ge}^{2}$, Steffen Renner ${ }^{2}$, Leandra Mansur ${ }^{2}$, Alicia Lindeman², Tayfun Tumkaya ${ }^{1}$, Carsten Russ ${ }^{2}$, Marc Hild ${ }^{2}$, Caroline Gubser Keller ${ }^{2}$, Jeremy L. Jenkins ${ }^{2}$, Kathleen A. Worringer ${ }^{1}$, Frederic D. Sigoillot ${ }^{2}$, Robert J. Ihry ${ }^{1}$

*These authors contributed equally to this work.

${ }^{1}$ Neuroscience, ${ }^{2}$ Chemical and Biological Therapeutics, Novartis Institutes for BioMedical

Research, Cambridge, MA

Correspondence: robert.ihry@novartis.com

\begin{abstract}
Unbiased transcriptomic RNA-seq data has provided deep insights about biological processes. However, its impact in drug discovery has been narrow given high costs and low throughput. Proof-of-concept studies with Digital RNA with pertUrbation of Genes (DRUG)-seq demonstrated the potential to address this gap. We extended the DRUG-seq platform by subjecting it to rigorous testing and by adding an open-source analysis pipeline. The results demonstrate high reproducibility and ability to resolve the mechanism(s) of action for a diverse set of compounds. Overall, the protocol and open-source analysis pipeline are a step towards industrializing RNAseq for high complexity transcriptomics studies performed at a saturating scale.
\end{abstract}




\section{Introduction}

In the pharmaceutical industry, it is standard to test thousands of compounds in highthroughput screens to identify regulators of a target or a biological process (Swinney and Anthony, 2011; Volochnyuk et al., 2019). This massive scale is made possible by focusing on a single readout. However, biological systems are inherently complex, and there is a need for scalable screening methods that can capture the total biological activity of small molecule libraries. Wholetranscriptome analysis, by RNA-seq, offers a high-dimensional readout but is cost prohibitive, and is typically performed on a small number of samples (Cleary et al., 2017; Verbist and Horchreiter, 2015).

To reduce costs, targeted RNA-seq approaches such as L1000 or RASL-seq have been deployed successfully as large-scale transcriptomic profiling methods ( $\mathrm{Li}$ et al., 2012; Subramanian et al., 2017). Targeted approaches such as these can be tailored to any gene set of interest, but it takes time to optimize gene sets for any given disease or cell model (Kong et al., 2021), and this approach may miss unexpected and potentially important transcriptomic signatures. Single cell RNA-seq can detect a few thousand genes per cell in an unbiased way. Recently the sci-Plex method identified cell-specific transcriptional responses to hundreds of compounds by labeling the cells in each treated well with a single-stranded DNA barcode that binds to nuclei prior to single cell (sc)RNA-seq sample processing, which enables sample multiplexing (Srivatsan et al., 2020). It will be promising to apply methods like sci-Plex to study the single cell effects of treatment in complex tissue models, such as brain organoids (Lancaster and Knoblich, 2014). As cell diversity increases, 1000s of cells must be profiled to detect responses in lower abundance or rare cell types. For more homogeneous cell culture models, scRNA-seq plus perturbation methods may offer less of an advantage over bulk RNA-seq methods and cellular resolution comes at the expense of throughput of perturbations tested and the number of genes detected.

DRUG-seq is a low-cost, high throughput bulk RNA-seq method that uses a direct in-well lysis of cells in 384-well plates and is ideal for studying the transcriptomic effect of many compound treatments in parallel (Ye et al., 2018). Multiple groups have been working to develop unbiased whole transcriptome-wide methods that are lower cost (Bush et al., 2017; Sholder et al., 2020; Yeakley et al., 2017). The pace of development in this field makes it difficult to compare data or perform exhaustive benchmarking studies to compare performance of the methods. As a key step toward this, we created an experimental design to thoroughly test the DRUG-seq platform and made the methods, data, and code available for independent verification and reproduction of the results. By exhaustively testing reproducibility across batches and plates, we demonstrate that DRUG-seq provides the granularity to bin compounds by mechanism of action (MoA) and meets the performance standards required of an industry-scale RNA-seq platform.

\section{Results}

\section{Summary of Protocol and Experimental Design}

DRUG-seq pairs high-throughput cell culture with miniaturized RNA-seq (Figure 1A). Cells are first cultured in 384-well plates and then treated with compounds for a desired period of time. After the treatment, the cells are directly lysed in each well, without RNA purification. The RNA in each well is used as a template for reverse transcription (RT), where the DRUG-seq RT primers 
incorporate both the well barcodes and unique molecular identifiers (UMI) (Figure 1B). After RT, the samples are pooled and used as a template for second strand cDNA synthesis and subsequent library construction. During library construction, Tn5-mediated cDNA tagmentation is performed which enzymatically fragments and adds Illumina adaptors to each insert. Next platelevel barcodes are incorporated to track multiple plates simultaneously. Finally, for optimal sequencing, libraries are size-selected, and quality checked by DNA fragment analysis. After quantification, libraries for each plate(s) are normalized and pooled, such that we can accurately target sequencing at a read depth equivalent to 1 million reads per well on the Illumina platform. The final product is 3' biased; full-length transcripts are not sequenced. The protocol scale can be adapted depending on the experimental need and resources available.

We designed an experiment to evaluate the reproducibility of the DRUG-seq protocol (Figure 2A). We generated 3 independent batches of $U-2$ OS cells on different days, plated in 3 replicate 384-well plates per batch for a total of 9x 384-well plates. In each plate, we treated cells with 14 compounds (Figure 2B, File S1, Table 1), each with an 8-point dose response (3.2 nM to $10 \mathrm{uM}$ ) with 3 replicates for each dose. This design was used to test the performance between batches of cells and plate(s).

\section{DRUG-seq Analysis Pipeline and Activity Threshold}

The data processing and primary analysis pipeline is a series of manually run steps, which allow for stepwise review and quality control. After sequencing, reads are demultiplexed using Illumina's standard bcl2fastq2 method. The upstream DRUG-seq data processing pipeline employs highly parallelized mapping, as well as barcode and UMI counting and filtering to efficiently generate count tables (Figure 3A). Mapping of sequencing reads is performed using a custom version of the Ensembl GRCh38 reference, in which each gene was constructed using all annotated exons.

The following steps of the analysis code are shared through GitHub (https://github.com/Novartis/DRUG-seq). Before generating differential expression (DE) results, an activity threshold is empirically determined based on the technical noise of the experiment and is coined the 'true null calculation'. This takes advantage of the high number of DMSO wells that can be sampled in this low-cost RNA-seq assay. The baseline for transcriptionally active treatments is set by performing multiple permutations of randomly sampled DMSO vs. DMSO comparisons using all available wells (step one). In step one, for each batch of three plates, three random DMSO wells were selected (one per plate) and were compared to the remaining 69 DMSO wells to then calculate DE. This process was iterated 500 times (Figure 3B, 4A, File S2). It should be noted that the number of iterations, as well as number of DMSO wells chosen as optimal reference controls can be customized for each experiment. Out of the 500 iterations, we identified both DMSO wells that contributed to the fewest DE genes and DMSO wells that contributed to the most DE genes using the Redundant siRNA activity (RSA) statistic (König et al., 2007). The nine best DMSO wells per batch (three per plate) were then used as reference controls to calculate DE for the compound treated samples (Figure 3B). DE analysis is conducted using limma-trend (Ritchie et al., 2015) to quantitate gene level changes in the transcriptome, which can serve as the input for additional analyses.

In step two, DMSO wells that potentially inflate the number of DEGs were removed ('bad' wells marked red, Figure 4A). The true null was recalculated by comparing three random DMSO 
wells versus the nine best DMSO wells with 500 iterations, where we selected the three random wells from the remaining DMSO wells (Figure 3C, 4A, File S3). When comparing the results from step one and two, the frequency of DMSO to DMSO comparisons yielding more than $100 \mathrm{DE}$ genes was reduced with reciprocal gains in the frequency of DMSO comparisons with 20 or fewer DE genes (Figure 4B). We typically define transcriptionally active compounds as those with more DE genes than the $95^{\text {th }}$ percentile of the DMSO to DMSO true null distribution (Figure $3 \mathrm{C}, 4 \mathrm{C}$ ). This indicates the treatment is more active than DMSO $95 \%$ of the time. Results obtained with all DMSO wells (Step one: Active > 221 DEGs) have a higher number of DEGs (Figure 4C). Results obtained by removing outlier DMSO wells (Step two: Active > 84 DEGs), lowered the minimal number of DEGs to be considered active (Figure $4 \mathrm{C}$ ).

For the secondary analysis pipeline discussed below, the step two true null threshold to filter active samples. The secondary analysis pipeline integrates count table data and experimental metadata for quality control and batch correction analyses. In addition to the true null (step two), gene-level thresholds are applied to reduce technical variation. Dimensionality reduction analysis by Uniform Manifold Approximation and Projection (UMAP) (Becht et al., 2019) is then performed to globally visualize the data.

\section{Batch and Plate Reproducibility Experiment with 14 Tool Compounds}

After using the true null threshold to select for active treatments, we used the secondary analysis pipeline and generated a UMAP from the DRUG-seq UMI counts matrix to visualize the global relationship between the 14 compound treatments across an active dose range (Figure 5A). After labelling the UMAP by either Louvain cluster number (Blondel et al., 2008) or compound treatment, it was evident that DRUG-seq identified many transcriptional groups. The 13 Louvain clusters were spatially distinct in the UMAP plot and often displayed substructures in the UMAP projection. We next examined how each compound was distributed across the UMAP and identified that the majority (12 of 14) of compounds were localized in a compound-specific cluster (Figure 5B, File S4, interactive plot). Dilazep and (S)-Crizotinib, were the exception, as these two compounds clustered near DMSO, suggesting low activity in the U2-OS cell line. Some compounds exhibited a single cluster at a certain dose range like BLU9931, CPI-203, CHIR118637 and Cmp_341. For most compounds, dose determined the clustering and we observed dose-specific clusters for Triptolide, Homoharringtonine, Brusatol, NVS-SM2, Fedratinib, Cmp_334 and AZD8055. These compounds may exhibit polypharmacology, and the targets they engage either change and/or interact across doses. Validation would be required to confirm these multi-targeted transcriptional activities. We observed co-clustering of compounds with related MoAs. For example, the translational inhibitors Homoharringtonine and Brusatol exhibited matched dose-dependent clustering, which reflects similar potencies and targets. Cmp_334, a PI3K inhibitor, and AZD8055, an mTOR inhibitor, exhibit a shared cluster at low doses that diverges with increasing concentration. This is not surprising given that PI3K is upstream of mTOR (Hay and Sonenberg, 2004) and perhaps both compounds have selective ontarget effects at the lower doses, while a broader range of scaffold-related activity is induced at higher doses. In addition to this descriptive analysis, we quantified cluster composition across batches. We used the Louvain method to define 13 clusters and generated a heat map to indicate the proportion of each active compound treatment within the Louvain clusters per each batch (Figure 5C). Each compound exhibits an enrichment in a specific Louvain cluster and the same 
result was observed across the 3 batches of cells. This indicates high reproducibility despite both technical and biological variation. Overall, these results indicate that DRUG-seq has sufficient resolution to group compounds by MoA, and because it is target agnostic, it can detect many MoAs in a single assay.

Next, the reproducibility of single compounds across doses was examined. Comparing a single dose of Homoharringtonine at $10 \mathrm{uM}$ across all 3 batches revealed an average overlap of $68 \%$, which is close to the expected overlap with a false discovery rate threshold of 0.1 (Figure $6 \mathrm{~A})$. Homoharringtonine exhibited a dose response and high overlap of DEGs across consecutive doses (Figure 6BC). Within a batch, adjacent doses 1, 3.16, and $10 \mathrm{uM}$ exhibited an average of $82 \%$ overlap in differentially expressed genes (Figure $6 \mathrm{C}$ ). We performed pairwise comparison across all doses and identified a high Pearson's correlation between samples within a batch. Higher doses and adjacent doses exhibited the highest correlations (.8-.98) (Figure 6D). We generated similar plots for the remaining 13 compounds and observed similar trends in active compounds that clustered away from DMSO. Overall, these studies represent a high bar for vetting the stability of a platform and will allow for comparisons with emerging technologies. We deposited the NGS data, metadata and analysis code for this study to enable other teams to reproduce the transcriptome signatures and to use in additional benchmarking studies. 


\section{Discussion}

DRUG-seq is a target-agnostic high-throughput screening method with a transcriptome readout, and it can be broadly applied to new cellular models without redesign of the approach or a priori assumptions about key genes or pathways that will be measured. It is well suited for high complexity RNA-seq studies in which many variables and perturbations are tested, such as the dose and length of treatment. DRUG-seq is a bulk RNA-seq readout, and, as such, is best applied to cell cultures with moderate to low heterogeneity. The bandwidth of DRUG-seq accommodates the profiling of a chemical series of related or unrelated chemotypes with different potencies and known or unknown on- and off-target activities. The total cost of DRUG-seq is \$3-10 per condition including triplicates at read depth of 0.25 to 1 million reads per well, respectively. This makes it possible to screen thousands of conditions while still providing a high-dimensional readout with greater than 7,000 genes. The resulting high-dimensional data can be used to group compounds by MoA, conduct user-defined signature queries, or search for compounds that may reverse disease signatures. Although the work reported here describes the usage of compounds, one can leverage DRUG-seq profiling for other perturbagens, depending on the question and biological model.

The low cost allowed us to systematically test both the technical and biological variability across plates and batches of cells. By standardizing the experimental design, performance metrics can be tracked long term across many experiments. Using this information, we set statistically defined thresholds to determine the activity of treatments tested in a DRUG-seq experiment. The true null threshold allows us to pick treatments that are statistically defined as active and provides a minimal range of DE genes, which we can trust to produce a reliable signature of expression. We also demonstrated that the results were highly reproducible across plates and batches of cells. The open-source analysis pipeline and available data will facilitate future analytical improvements and lower the barrier for new labs to adopt the platform.

By deploying transcriptomics at scale, we stand to gain biological insights beyond a single target or pathway. With the selection of a set of compounds with diverse MoAs, we demonstrated the granularity of DRUG-seq to discern specific MoAs, dose responses and dose-dependent polypharmacology. This would be easy to miss if only a single or a few doses were tested. DRUGseq is unbiased, as selection of panels of genes is not required, and it quantifies 5 to $10 x$ more transcripts than L1000 or other targeted amplicon approaches (Li et al., 2012; Subramanian et al., 2017). The wide range of activity detected by DRUG-seq allows it to detect expected and unexpected biological responses. Being blind to the latter has likely contributed to the failure of many drugs at various stages of discovery and development. Furthermore, the dose-dependent detection of on- and off-target phenotypes and the switch between phenotypes can be used to determine potencies for each effect (Renner et al., 2020) and potentially quantify selectivity and safety windows in the absence of dedicated assays. 
A
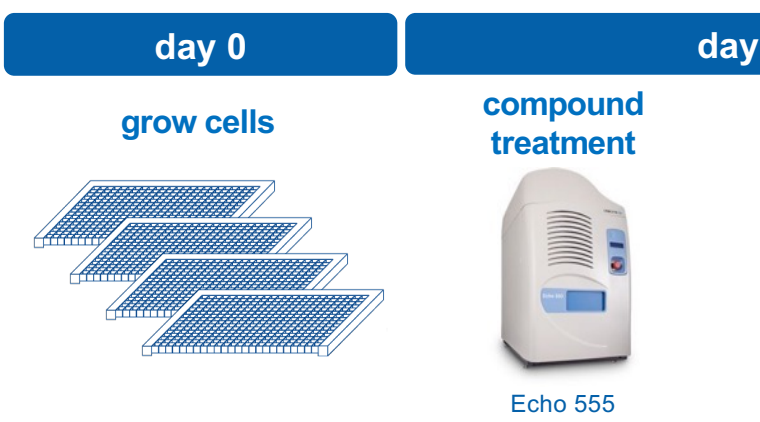

day 1

cell lysis \&

freeze at $-80 \mathrm{C}$

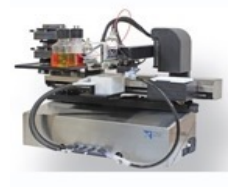

GNF W/D

day 4

quantification

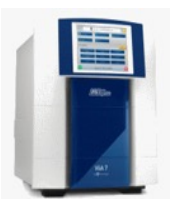

qPCR

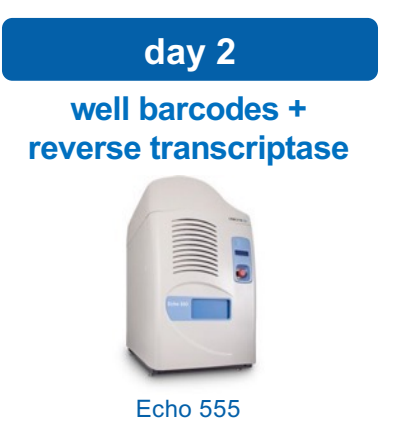

day 5

sequencing

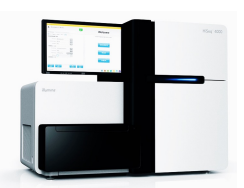

Illumina sequencer

B

Day 1: Direct in-well lysis:

5'-mRNA न IIIIIIIIIIIIIIIIIII - -3'

Day 2: Reverse Transcription (A), Template Switch (B) and 384 well Pooling:

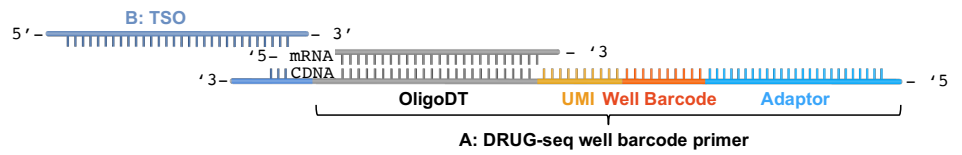

Day 3: Library Construction (Tagmentation (A), Amplification (B)):

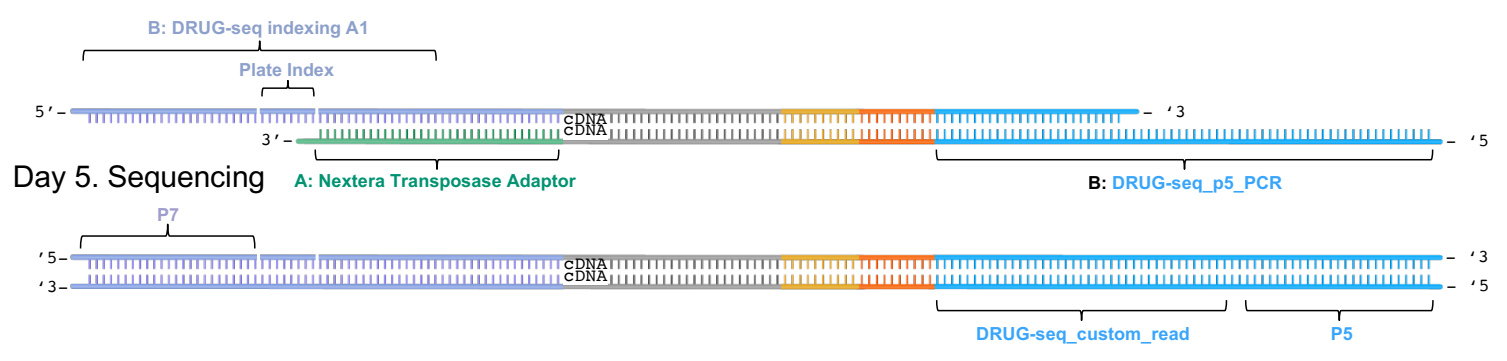

Figure 1. Overview of the DRUG-seq protocol. (A) Once cells are plated and treated with perturbagens the DRUG-seq protocol can be performed and sequenced in 5 days. The process and equipment depicted here is for batches of 18 or fewer plates. Scale up or down is possible depending upon infrastructure available. (B) Depiction of the DRUG-seq high throughput RNA-seq chemistry (Adapted from Ye et. al., 2018). Day 1: The scale of DRUG-seq is enabled by using a direct in-well lysis of cells without mRNA purification, which is coupled to a barcoding and sequencing strategy, on day 2, that tracks transcripts (UMI), wells (well barcode), and plates of cDNA libraries (Illumina Nextera or custom Illumina indexes) sequenced on the Illumina platform (days 3-5). 
A

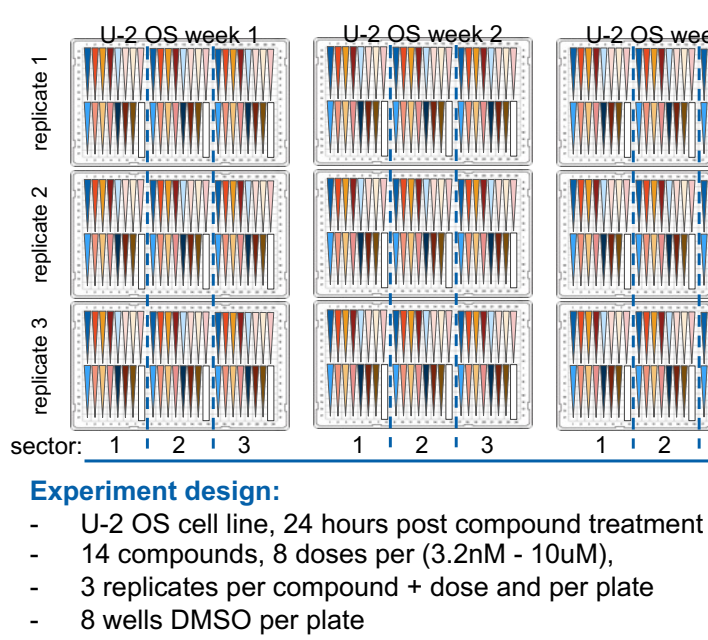

B

\begin{tabular}{|c|c|c|c|}
\hline Compound & NSN identifier & Target & $\begin{array}{l}\text { Cluster } \\
\text { Ye et al. }\end{array}$ \\
\hline Triptolide & ND09-QY33 & ERCC3i & IV \\
\hline CHIR118637 & CA-90-VK59 & GSK3Bi & \\
\hline Cmp_341 & JC-68-AL90 & JAK2i & IV \\
\hline Fedratinib & MB-03-IE36 & JAK2i & III \\
\hline NVS-SM2 & JC-43-ZO95 & SNRPCmod & II \\
\hline CPI-203 & PA-33-CK45 & BRD4i & III \\
\hline Brutasol & KA-73-NB69 & NRF2i & VI \\
\hline Homoharringtonine & QC-05-UB63 & EIF4Ei & VI \\
\hline BTdCPU & SE-15-AV21 & EIF2AK1a & VI \\
\hline AZD8055 & NV-67-DX31 & MTORi & VI \\
\hline Cmp_334 & DB-85-YA47 & $\mathrm{PI} 3 \mathrm{~K}$ & 1 \\
\hline Dilazep & NA-37-JQ34 & SLC29A2i & \\
\hline BLU9931 & VA-76-OV33 & FGFR4 & IV \\
\hline (S)-Crizotinib & LD-22-SA99 & ALK & IV \\
\hline
\end{tabular}

Figure 2. DRUG-seq reproducibility experimental design. (A) Experimental design depicted by 9 plate maps. Within each plate 14-compounds were plated in 8-doses (3.2nM-10uM) in 3-sectors for a total of 3 replicates per condition per plate ( $n=3 /$ per condition). Each sector contained 8 DMSO wells for a total of 24 per plate $(n=24)$. For each week (or batch) of cells there were 3 replicate plates. Each batch of cells were plated on independent days to reflect biological variability. (B) Table depicts compound name, identifier, target, and cluster from Ye et. al., 2018 publication. 14-compounds were selected to represent a diverse set of MoAs. 


\section{A. Genome mapping and counting of raw NGS data}

1. Bcl files converted to fastq

2. Fastq decompression and STAR alignment with Ensembl GRCh38 reference

3. Generate read (unfiltered) and UMI (filtered) counts for well and plate indexes

B. Generating Differential Expression Results

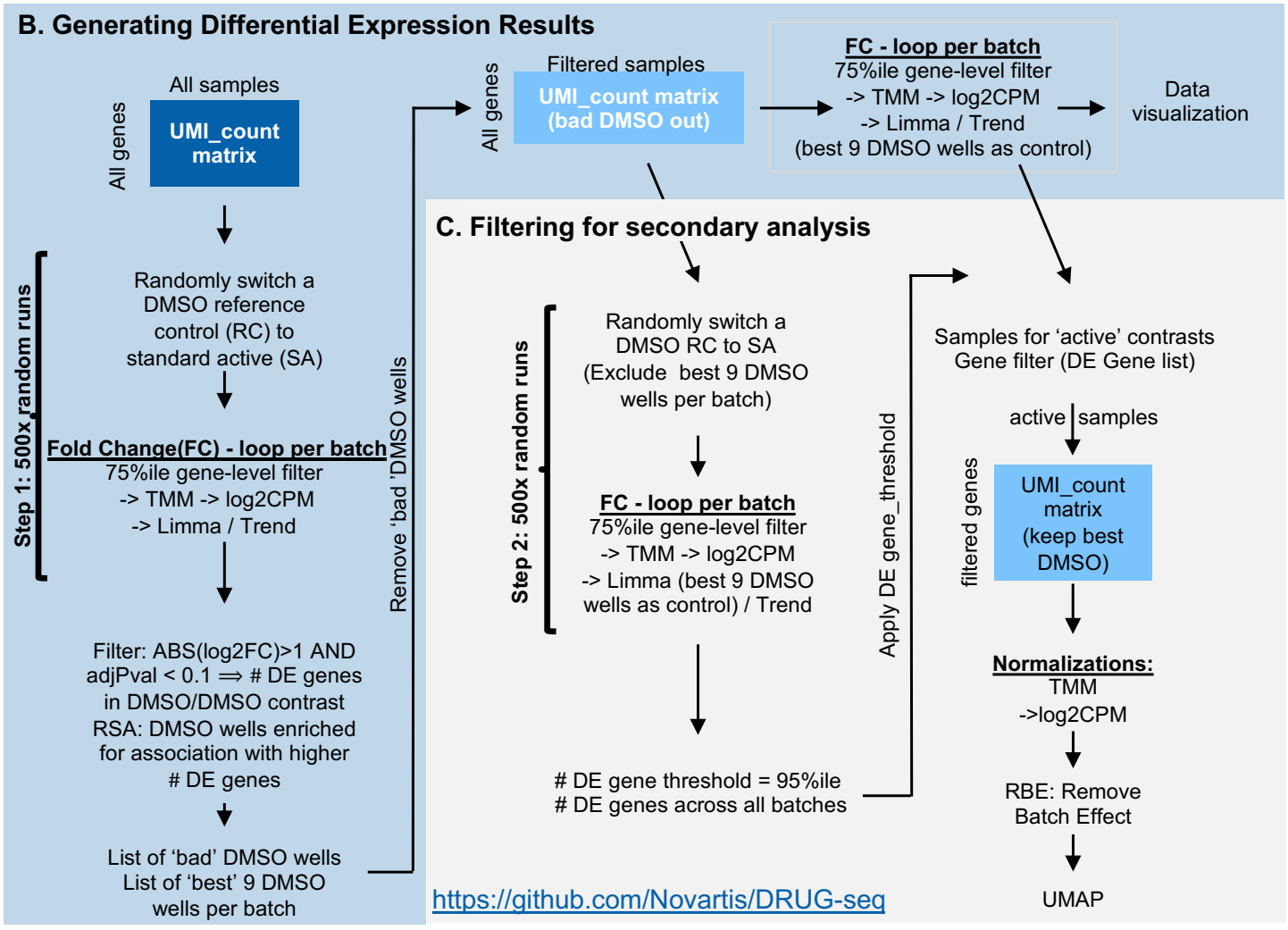

Figure 3. DRUG-seq analysis pipeline. The DRUG-seq analysis pipeline is comprised of 3 steps (A) Outline of the steps required to convert raw next generation sequencing data into a count matrix by converting to fastq files, aligning to the transcriptome and counting transcripts associated with well and plate barcodes. (B) Flow chart describing how differential expression results are obtained from the UMI count matrix generated in part A. In step one, the true null is calculated by performing 500 random DMSO to DMSO comparisons to generate DE results. Next, RSA analysis is used to rank DMSO wells by the number of DE genes they contributed to. Bad DMSO wells are removed and the 9 best DMSO wells are used as reference control (RC) to calculate differential expression for the compound treated wells. (C) Flow chart describing the filtering steps required for secondary analysis. Step two of the true null calculation is performed and 500 random DMSO to DMSO comparisons generate DE gene results in the absence of bad wells. The $95^{\text {th }}$ percentile of the 500 -comparison distribution is then used as an activity threshold. The threshold is the minimal number of DE genes required to be greater than the technical noise in DMSO 95\% of the time. The true null activity threshold is used to filter active samples. DE gene filtering, normalization, and removal of batch effects are applied to generate the final UMAP visualization. 
A typical design
(treatment v. DMSO)

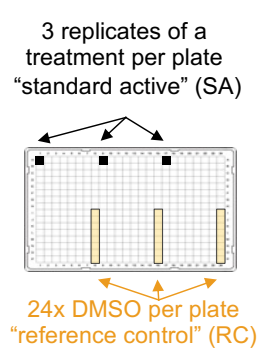

B

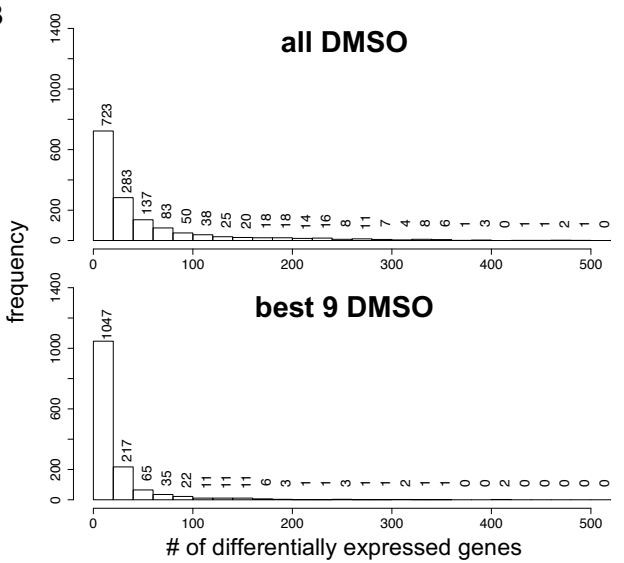

true null - step 1 (3 DMSO v. 69 DMSO)

3 random replicates of DMSO to

SA per batch

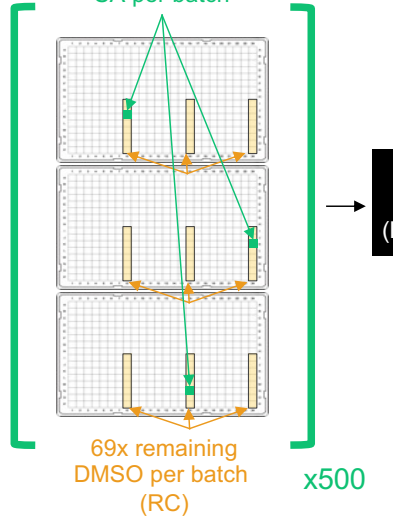

C

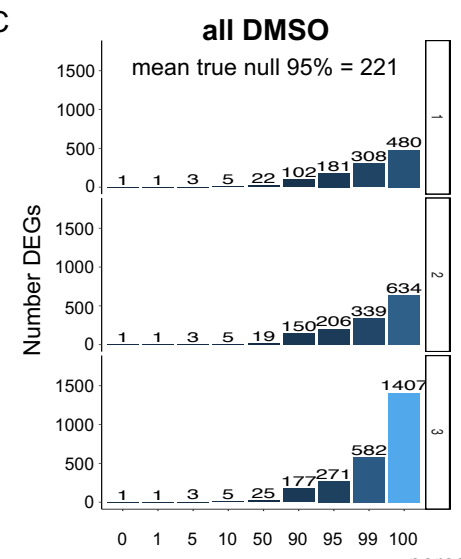

true null - step 2

(3 DMSO v. 9 best DMSO)

3 random replicates of DMSO to SA per batch

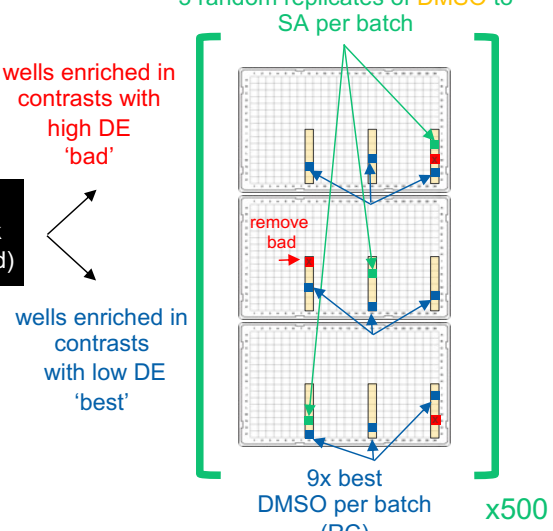

(RC)

best 9 DMSO wells

mean true null $95 \%=84$

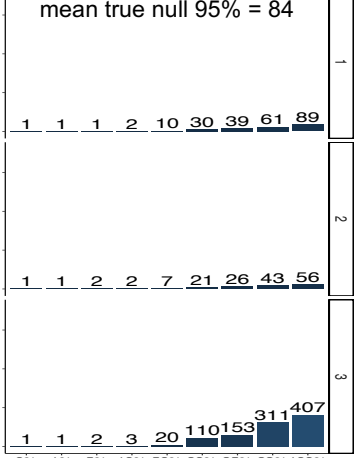

$\%$

Figure 4. DRUG-seq activity threshold set by the true null calculation.

(A) The left panel depicts the typical analysis of a DRUG-seq experiment using compounds with DMSO as a control. When setting a contrast for DE analysis, 3 replicates of a standard active (SA) sample (compound plus dose, colored black) are compared to the DMSO Reference Controls (RC, colored gold)). Each compound treatment has 3 SA well replicates and 24 RC or DMSO well replicates per plate. The middle panel depicts step 1 of the true null calculation. For this, the number of differentially expressed genes is quantified when comparing $3 \mathrm{DMSO}$ well replicates as mock treatments (DMSO/RC turned SA wells, green) relative to the remaining $69 \mathrm{DMSO} / \mathrm{RC}$ (gold) wells per batch of 3 plates. 500 randomly chosen differential expression comparisons of 3 DMSO versus the remaining 69 DMSO are performed. Next outlier DMSO wells (colored red) and the best DMSO wells (colored blue) are identified using the Redundant siRNA Activity (RSA) statistical ranking analysis. The right panel depicts step 2 of the true null calculation. 500 random DMSO to DMSO differential expression comparisons are recalculated, this time in the absence of the bad DMSO wells with the 9 best DMSO as the RC. (B) Histogram shows the frequency of the number of DE genes per comparison of randomized DMSO SA to DMSO RC comparisons. In step 1, all DMSO wells are compared (top) and in step 2, the bad DMSO wells are removed and the randomly chosen DMSO wells are compared to the best 9 DMSO wells (bottom). Y-axis is the frequency of the 500 DMSO comparisons, across 3 batches, binned by number of differentially expressed genes on the X-axis. (C) The bar graph plots the number DE genes (y-axis) against the percentile from distribution of 500 randomized DMSO to DMSO comparisons per batch of 3 plates. Left panel depicts true null calculated using all DMSO wells (step 1) and selected a mean threshold of 221 differentially expressed genes (DEG) at a 95 percentile. Depicted on the right panel is the true null calculation removing the outlier DMSO wells across 3 plates (mean 84 DEG at the 95th percentile). The result is interpreted as, only $5 \%$ of the time DEGs in DMSO are above the noise detected by comparing DMSO treatments. Removing the outlier DMSO wells for analysis lowers the threshold per batch. Light blue - 1407 DEGs and dark blue is 1 DEG. 
A

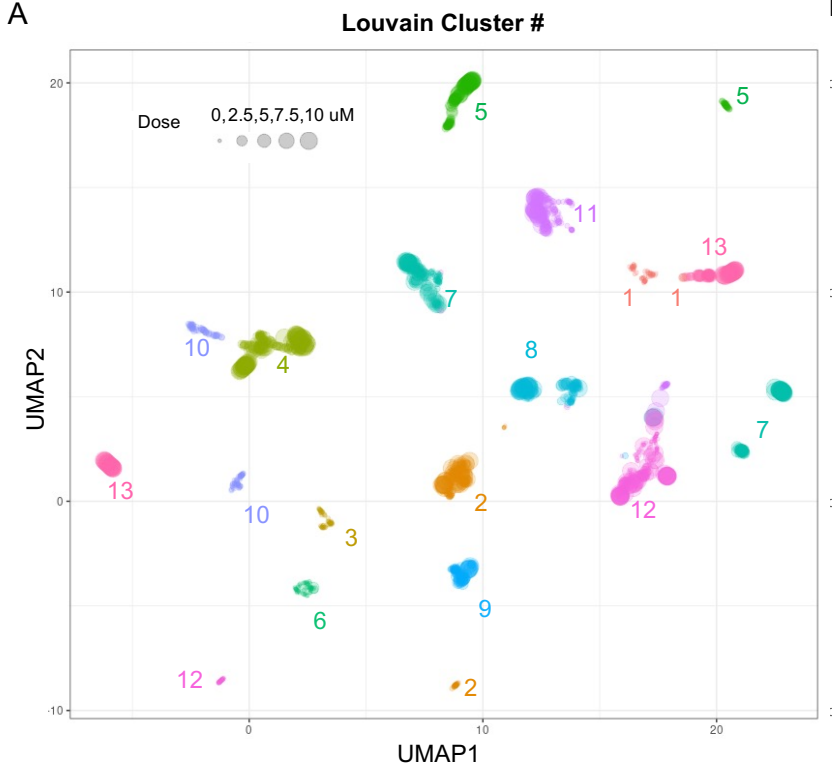

C

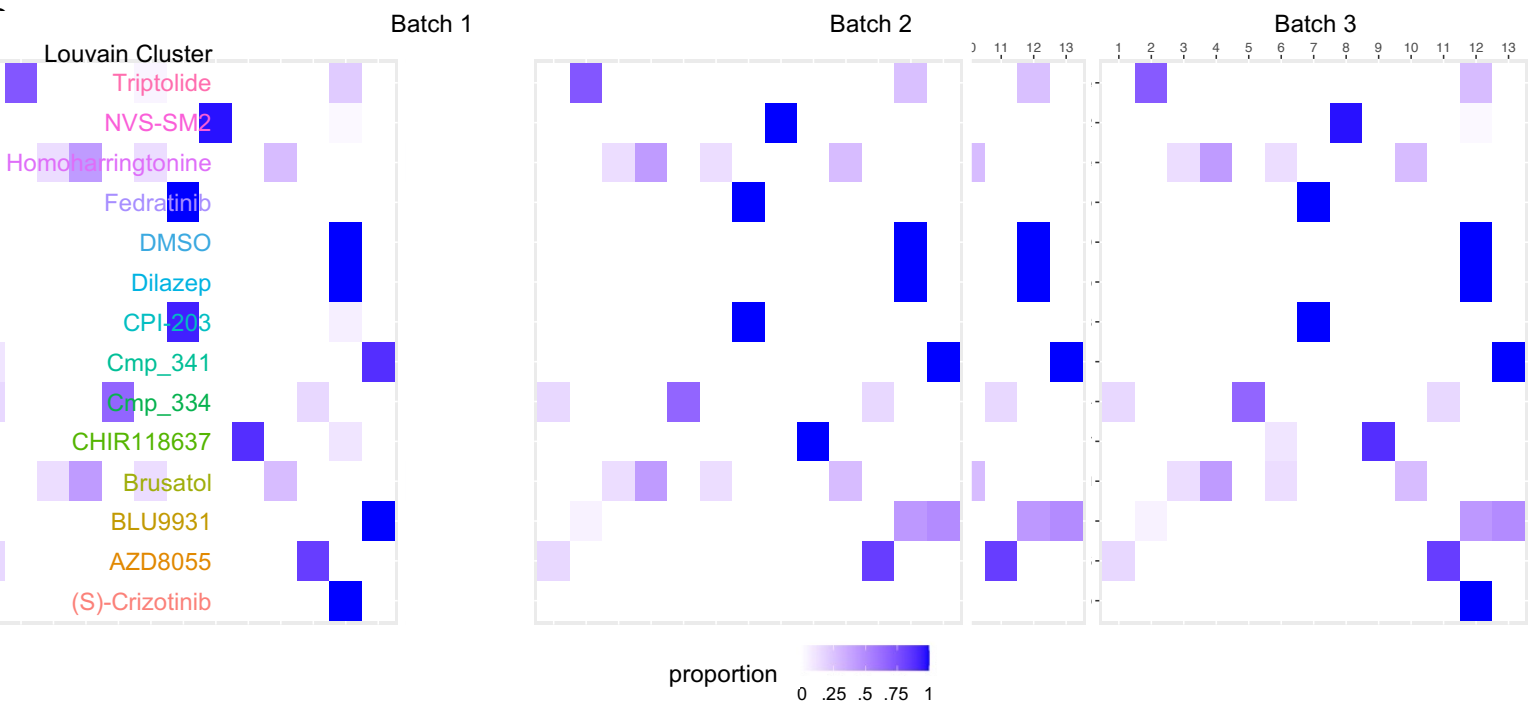

B

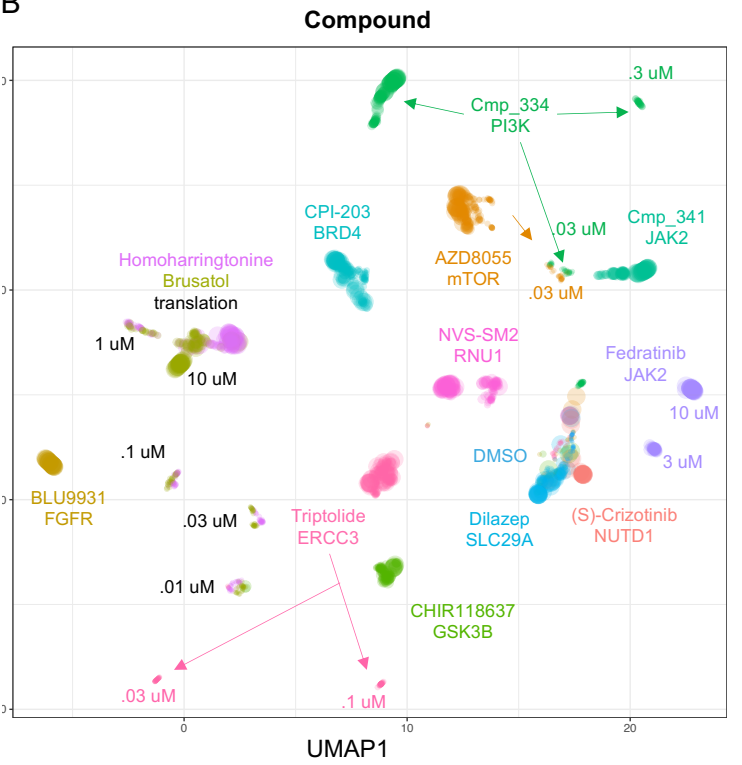

Batch 2 

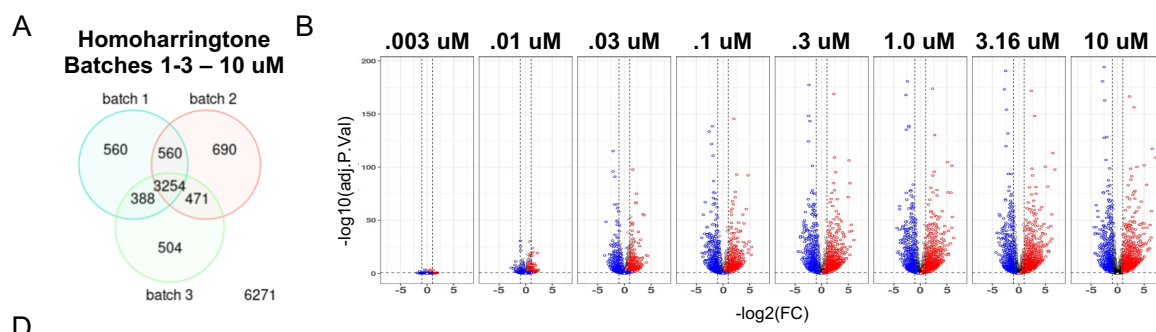

Homoharringtone
Batch 1 - 1-10 uM
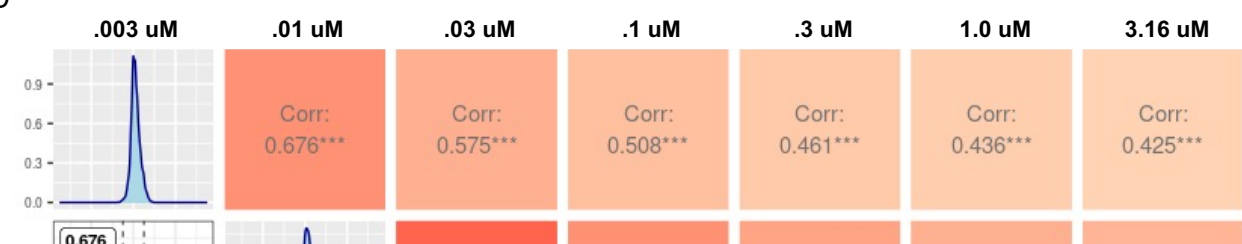

$1.00144 \mathrm{uM} \quad 10 \mathrm{uM}$

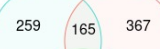

$244^{4060} 432$
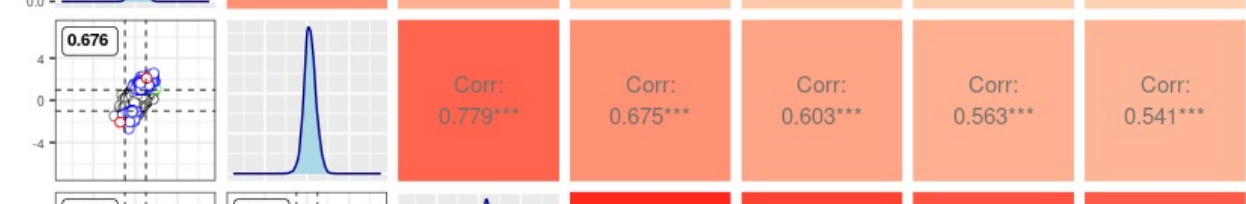

296

$3.16456 \mathrm{uM} \quad 8257$
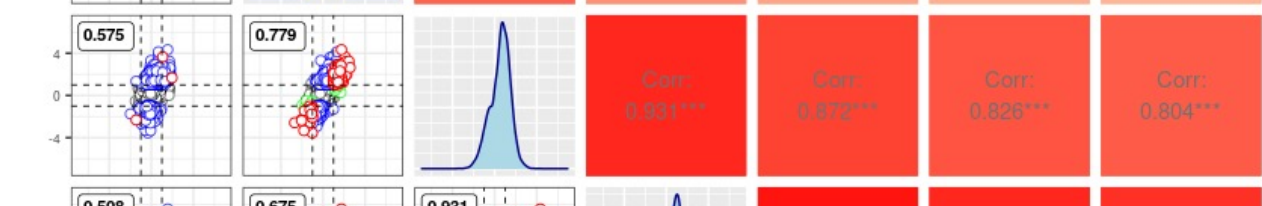

$10 \mathrm{uM}$
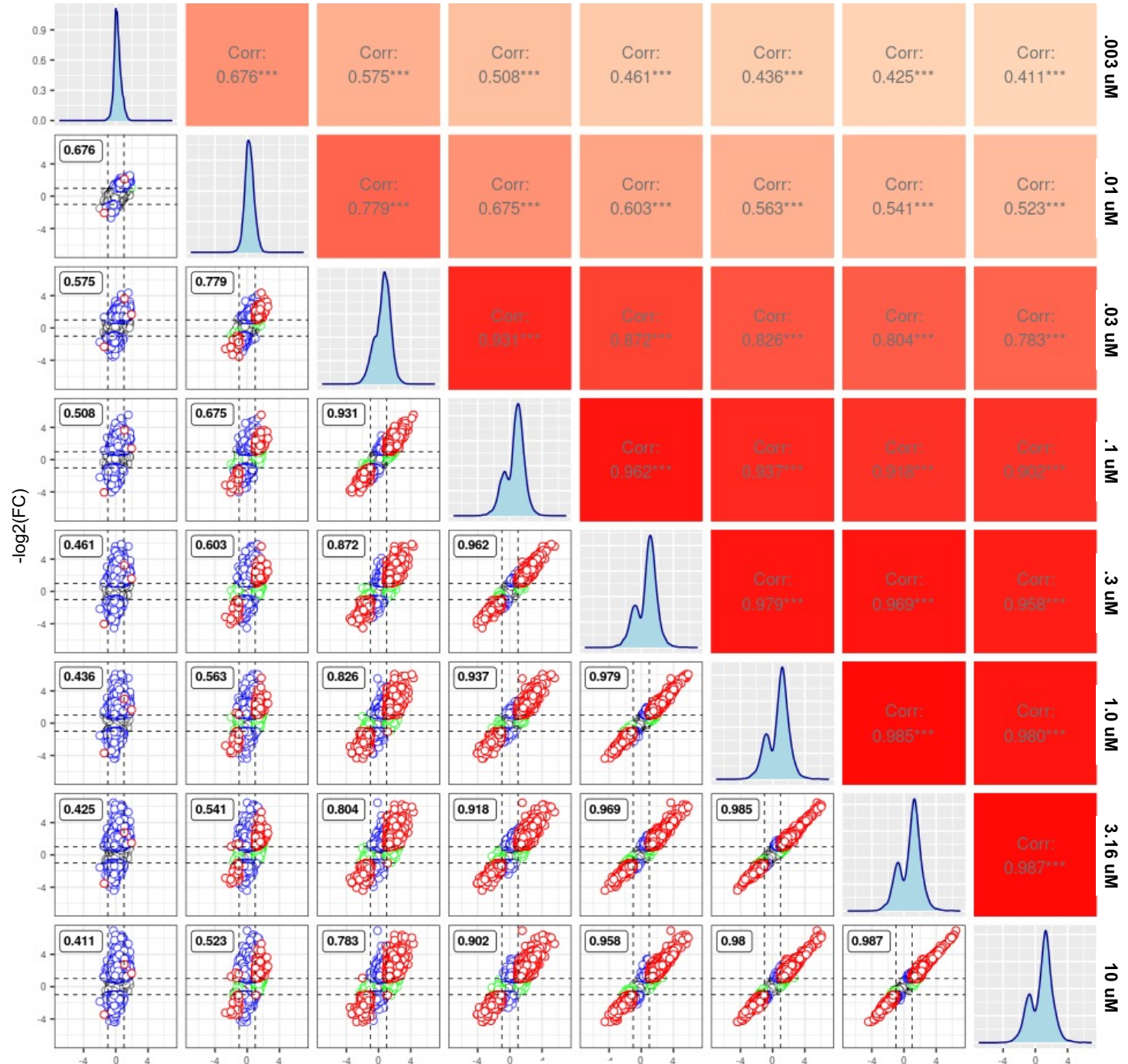

$-\log 2(\mathrm{FC})$

Figure 6. Dose response and batch reproducibility of Homoharringtonine. (A) Venn diagram showing overlapping differentially expressed genes across batches 1-3 at 10uM dose. (B) Volcano plots depicting dose-response for Homoharringtonine. Y-axis adjusted $p$-value, $X$-axis $\log 2(\mathrm{FC})$. Red $=$ upregulated genes $10 \mathrm{uM}$, Blue $=$ downregulated genes $10 \mathrm{uM}$, n.s. $=$ not significant in $10 \mathrm{uM}$ (significant $=$ adj. $p<0.5+/-1$ log2FC). (C) Venn diagram depicting overlap of differentially expressed genes between adjacent doses 110uM. (D) Pair plot comparing all doses. Top right half indicates Pearson's correlation between samples, color scale from white $=0$ to red $=1$. Bottom left scatter plots pairwise compare log2(FC) in gene expression from conditions labeled on the top and right edges. The dashed lines label log2(FC) threshold equal to 1. Red indicates common DEGs across conditions. Blue are DEGs specific to condition on y-axis and green are DEGs specific to the $x$-axis for each comparison. The histogram on the diagonal the depicts the distribution of gene expression for each condition. 
Table 1. 14-compounds

\begin{tabular}{cccc} 
Compound & NSN identifier & Target & $\begin{array}{c}\text { Cluster } \\
\text { Ye et. al., 2018 }\end{array}$ \\
Triptolide & ND-09-QY33 & ERCC3i & IV \\
\hline CHIR118637 & CA-90-VK59 & GSK3Bi & \\
\hline Cmp_341 & JC-68-AL90 & JAK2i & IV \\
\hline Fedratinib & MB-03-IE36 & JAK2i & III \\
\hline NVS-SM2 & JC-43-ZO95 & SNRPCmod & II \\
\hline CPI-203 & PA-33-CK45 & BRD4i & III \\
\hline Brutasol & KA-73-NB69 & NRF2i & VI \\
\hline Homoharringtonine & QC-05-UB63 & EIF4Ei & VI \\
\hline BTdCPU & SE-15-AV21 & EIF2AK1a & VI \\
\hline AZD8055 & NV-67-DX31 & MTORi & VI \\
\hline NVP-BVB808/Cmp_334 & DB-85-YA47 & PI3K & I \\
\hline Dilazep & NA-37-JQ34 & SLC29A2i & IV \\
\hline BLU9931 & VA-76-OV33 & FGFR4 & IV
\end{tabular}




\section{Acknowledgements}

We would like to thank Dan Palacios and Mark Healy for reviewing the compound lists. We would like to thank Ricardo Dolmetsch for support of the DRUG-seq platform. Thank you, Alan Abrams, for rendering figure $1 \mathrm{~B}$.

\section{Author Contributions}

D.J.H., C.Y., and M.H., developed the protocol and generated the 14-compound dataset. A.L. performed sequencing. L.M. tested the protocol as a first-time user and wrote methods. C.R. and M.H. designed the 14-compound experiment. C.R., M.H., C.G.K., K.A.W. and J.L.J. provided experimental feedback. J.L., F.D.S., R.G., M.N., S.R., and D.J.H., developed computational methods to analyze DRUG-seq data. T.T. tested the code as a first-time user. R.J.I., D.J.H., J.L. and K.A.W. wrote the manuscript.

\section{Competing Interests}

All authors are employees of the Novartis Institutes for BioMedical Research.

\section{Additional information}

File S1. Metadata for 14-Compound experiment

File S2. Random DMSO wells selected for Step 1

File S3. Random DMSO wells selected for Step 2

File S4. Interactive UMAP (Plotly)

Raw Data: GSE176150

Code: https://github.com/Novartis/DRUG-seq 


\section{References}

1. Becht, E., Mcinnes, L., Healy, J., Dutertre, C., Kwok, I.W.H., Ng, L.G., Ginhoux, F., and Newell, E.W. (2019). Dimensionality reduction for visualizing single-cell data using UMAP. Nat. Biotechnol. 37.

2. Blondel, V.D., Guillaume, J.-L., Lambiotte, R., and Lefebvre, E. (2008). Fast unfolding of communities in large networks. J. Stat. Mech. Theory Exp. 2008, P10008.

3. Bush, E.C., Ray, F., Alvarez, M.J., Realubit, R., Li, H., Karan, C., Califano, A., and Sims, P.A. (2017). PLATE-Seq for genome-wide regulatory network analysis of highthroughput screens. Nat. Commun. 8.

4. Cleary, B., Cong, L., Cheung, A., Lander, E.S., Regev, A., Cleary, B., Cong, L., Cheung, A., Lander, E.S., and Regev, A. (2017). Efficient Generation of Transcriptomic Profiles by Theory Efficient Generation of Transcriptomic Profiles by Random Composite Measurements. Cell 171, 1424-1436.e18.

5. Consortium, S.W.G. of the P.G., Ripke, S., Walters, J.T., and O'Donovan, M.C. (2020). Mapping genomic loci prioritises genes and implicates synaptic biology in schizophrenia. MedRxiv 2020.09.12.20192922.

6. Hay, N., and Sonenberg, N. (2004). Upstream and downstream of mTOR. Genes Dev. 18, 1926-1945.

7. Huang, Z., and Lippard, S.J. (2014). Tris(2-pyridylmethyl)amine (TPA) as a membranepermeable chelator for interception of biological mobile zinc†. Metallomics 5, 648-655.

8. Kong, L., Chen, Y., Xu, F., Xu, M., Li, Z., Fang, J., Zhang, L., and Pian, C. (2021). Mining influential genes based on deep learning. BMC Bioinformatics 22, 1-12.

9. König, R., Chiang, C., Tu, B.P., Yan, S.F., DeJesus, P.D., Romero, A., Bergauer, T., Orth, A., Krueger, U., Zhou, Y., et al. (2007). A probability-based approach for the analysis of large-scale RNAi screens. Nat. Methods 4, 847-849.

10. Lancaster, M. a., and Knoblich, J. a. (2014). Organogenesis in a dish: modeling development and disease using organoid technologies. Science 345, 1247125.

11. Li, H., Qiu, J., and Fu, X.D. (2012). RASL-seq for Massively Parallel and Quantitative Analysis of Gene Expression. Curr. Protoc. Mol. Biol. 1, 1-9.

12. Niu, H.M., Yang, P., Chen, H.H., Hao, R.H., Dong, S.S., Yao, S., Chen, X.F., Yan, H., Zhang, Y.J., Chen, Y.X., et al. (2019). Comprehensive functional annotation of susceptibility SNPs prioritized 10 genes for schizophrenia. Transl. Psychiatry 9.

13. Pan, E., Zhang, X., Huang, Z., Krezel, A., Zhao, M., Tinberg, C.E., and Lippard, S.J. (2011). Article Vesicular Zinc Promotes Presynaptic and Inhibits Postsynaptic LongTerm Potentiation of Mossy Fiber-CA3 Synapse. Neuron 71, 1116-1126.

14. Renner, S., Bergsdorf, C., Bouhelal, R., Koziczak-Holbro, M., Amati, A.M., TecherEtienne, V., Flotte, L., Reymann, N., Kapur, K., Hoersch, S., et al. (2020). Genesignature-derived IC50s/EC50s reflect the potency of causative upstream targets and downstream phenotypes. Sci. Rep. 10, 9670.

15. Ritchie, M.E., Phipson, B., Wu, D., Hu, Y., Law, C.W., Shi, W., and Smyth, G.K. (2015). limma powers differential expression analyses for RNA-sequencing and microarray studies. 43.

16. Sholder, G., Lanz, T.A., Moccia, R., Quan, J., Aparicio-Prat, E., Stanton, R., and Xi, H.S. (2020). 3'Pool-seq: An optimized cost-efficient and scalable method of wholetranscriptome gene expression profiling. BMC Genomics 21, 1-11.

17. Srivatsan, S.R., McFaline-Figueroa, J.L., Ramani, V., Saunders, L., Cao, J., Packer, J., Pliner, H.A., Jackson, D.L., Daza, R.M., Christiansen, L., et al. (2020). Massively multiplex chemical transcriptomics at single-cell resolution. Science (80-. ). 367, 45-51.

18. Subramanian, A., Narayan, R., Corsello, S.M., Peck, D.D., Natoli, T.E., Lu, X., Gould, J., Davis, J.F., Tubelli, A.A., Asiedu, J.K., et al. (2017). A Next Generation Connectivity Map: L1000 Platform and the First 1,000,000 Profiles. Cell 171, 1437-1452.e17. 
19. Swinney, D.C., and Anthony, J. (2011). How were new medicines discovered? Nat. Rev. Drug Discov. 10, 507-519.

20. Verbist, B., and Horchreiter, S. (2015). Using transcriptomics to guide lead optimization in drug discovery projects: Lessons learned from the QSTAR project. Drug Discov. Today 20.

21. Volgraf, M., Sellers, B.D., Jiang, Y., Wu, G., Ly, C.Q., Villemure, E., Pastor, R.M., Yuen, P., Lu, A., Luo, X., et al. (2016). Discovery of GluN2A-Selective NMDA Receptor Positive Allosteric Modulators (PAMs): Tuning Deactivation Kinetics via Structure-Based Design.

22. Volochnyuk, D.M., Ryabukhin, S. V, Moroz, Y.S., Savych, O., Chuprina, A., Horvath, D., Zabolotna, Y., Varnek, A., and Judd, D.B. (2019). Evolution of commercially available compounds for HTS. Drug Discov. Today 24, 390-402.

23. Working, S., and Consortium, G. (2014). Biological insights from 108 schizophreniaassociated genetic loci.

24. XiangWei, W., Jiang, Y., and Yuan, H. (2018). De novo mutations and rare variants occurring in NMDA receptors. Curr. Opin. Physiol. 2, 27-35.

25. Ye, C., Ho, D.J., Neri, M., Yang, C., Kulkarni, T., Randhawa, R., Henault, M., Mostacci, N., Farmer, P., Renner, S., et al. (2018). DRUG-seq for miniaturized high-throughput transcriptome profiling in drug discovery. Nat. Commun. 9.

26. Yeakley, J.M., Shepard, P.J., Goyena, D.E., Vansteenhouse, H.C., McComb, J.D., and Seligmann, B.E. (2017). A Trichostatin a expression signature identified by TempO-Seq targeted whole transcriptome profiling. PLoS One 12.

27. Zhang, Y., Pak, C., Han, Y., Ahlenius, H., Zhang, Z., Chanda, S., Marro, S., Patzke, C., Acuna, C., Covy, J., et al. (2013). Rapid single-step induction of functional neurons from human pluripotent stem cells. Neuron 78, 785-798. 


\section{Materials and Methods}

Day 1: Cell Culture

U2-OS (ATCC HTB-96) were grown in DMEM, 10\% FBS, and 1\% Pen/Strep. Sufficient number of cells were grown prior to trypsin dissociation the day of plating. 20 ul of cells were dispensed into 384-well black uClear polystyrene cell culture-treated plates (Griener, Cat\#: 781090) using a bottle valve washer/dispenser from the Genomics Institute of the Novartis Research Foundation (GNF, http://www.gnfsystems.com) with a concentration of 5000 cells per well, a day prior to compound treatment. The GNF system is critical for large-scale experiments, but other standard plate wash/dispense equipment or multichannel pipettes will suffice for smaller scale. Density optimization is required for each cell line for optimal downstream steps.

Ngn2 neurons were generated by exposing transgenic H9-hESCs with a dox inducible Ngn2 to doxycycline (1.9 ug/mL) for 3 days in DMEM F12 Glutamax 95\%, B27 2\%, Pen/Strep 1\% and N2 1\%. Immature neurons were dissociated using accutase. Ngn2 neurons were then replated in matrigel coated plates at a density of 12,000 per well in 80ul of media (DMEM F12 Glutamax 95\%, B27 2\%, Pen/Strep 1\%, N2 1\%, NT3 $9.5 \mathrm{ng} / \mathrm{mL}, 3.8 \mathrm{ng} / \mathrm{mL}$ BDNF) with doxycycline $(1.9 \mathrm{ug} / \mathrm{mL})$. The Ngn2 neurons were hemi-fed every other day until compound treatment $(24 \mathrm{~h}$ start at day 13$)$.

Day 1-2: Compound Treatment and Lysis

\begin{tabular}{|l|c|}
\hline \multicolumn{2}{|l|}{ 2x Lysis Buffer Stock - store at room temp } \\
\hline Component & Volume \\
\hline $1 \mathrm{M}$ Tris-HCl pH8.0 (Life Tech,15568-025) & $1000 \mathrm{ul}$ \\
\hline $2 \mathrm{M} \mathrm{KCl}($ Ambion, AM9640G) & $750 \mathrm{ul}$ \\
\hline $20 \%$ of $20 \%$ Ficoll PM-400 in H2O (Sigma, F5415-25ML) & $6000 \mathrm{ul}$ \\
\hline Triton-100 (Sigma, T8787-100ML) & $30 \mathrm{ul}$ \\
\hline $\mathrm{H}_{2} \mathrm{O}$ & $2220 \mathrm{ul}$ \\
\hline
\end{tabular}

\begin{tabular}{|l|c|}
\hline \multicolumn{2}{|l|}{ 2x Lysis Buffer - make fresh each day } \\
\hline Component & Volume \\
\hline 2x Lysis Buffer Stock & 4800 ul \\
\hline RiboLock RNase Inhibitor 40U/ul (ThermoFisher, EO0381) & 120 ul \\
\hline
\end{tabular}

One day after plating, $20 \mathrm{~nL}$ of each compound were added using an acoustic dispense Echo 555 Liquid Handler (Labcyte). After a 12 to 24-hour compound treatment, the media was aspirated down to $7.5 \mathrm{ul}$ and an equal amount of $2 x$ lysis buffer was added to all wells using the bottle valve washer/dispenser from GNF (U2-OS Plates were sealed and placed on a microplate shaker HT-91002 (BigBear Automation) for 4 minutes (min) at 900 rpm. Lysis duration is cell typeand density-dependent and requires optimization. Ngn2 neurons plates were lysed for 8 minutes. Plates were then centrifuged at $2000 \mathrm{rpm}$ for $1 \mathrm{~min}$ before storage at $-80 \mathrm{C}$ until ready for further sample processing. Plates can be stored in $-80 \mathrm{C}$ for up to 3-4 weeks, after which RNA quality may begin to deteriorate.

\section{Day 3: Reverse Transcription and Library Construction}

\begin{tabular}{|l|c|}
\hline RT Mix \\
\hline Component & per well \\
\hline 5XRT Buffer (ThermoFisher, EP0742) & 0.5 ul \\
\hline
\end{tabular}




\begin{tabular}{|l|l|}
\hline $1 \mathrm{M} \mathrm{MgCl}_{2}$ (Ambion, AM9530G) & $0.1 \mathrm{ul}$ \\
\hline Regular template switching primer 100 uM (Integrated DNA Technologies) & $0.1 \mathrm{ul}$ \\
\hline RiboLock RNase Inhibitor 40U/ul (ThermoFisher, EO0381) & $0.1 \mathrm{ul}$ \\
\hline dNTP 2.5 uM (ThermoFisher, AM8228G) & $0.1 \mathrm{ul}$ \\
\hline Maxima RT (ThermoFisher, EP0742) & $0.1 \mathrm{ul}$ \\
\hline $\mathrm{H}_{2} \mathrm{O}$ (ThermoFisher, AM8228G) & $1.5 \mathrm{ul}$ \\
\hline
\end{tabular}

On the day of sample processing, the assay plates were placed on ice until thawed. In an Armadillo PCR plate (ThermoFisher, AB2384), 2.75 ul of RT mix was added using a Multidrop Combi (ThermoFisher, 5840300). Once the assay plates were thawed, 15 ul of cell lysate was transferred using a Bravo (Agilent Technologies) into the plate containing RT mix. $10 \mathrm{nl}$ of $1 \mathrm{uM}$ barcoded DRUG-seq RT primers were then dispensed into each well using an Echo 555 Liquid Handler (Labcyte). Plates were sealed (BioRad, MSB1001), centrifuged for $1 \mathrm{~min}$ at $2000 \mathrm{rpm}$ and incubated at $42 \mathrm{C}$ on a ProFlex PCR system (ThermoFisher, 4484077) for 2 hours.

After RT, samples were centrifuged for $1 \mathrm{~min}$ at $2000 \mathrm{rpm}$. Next, each individual plate was pooled into a reagent reservoir (ThermoFisher, 1064-05-7) using a Bravo Automated Liquid Handling Platform (Agilent). Samples were then transferred from the reservoir into a $50 \mathrm{~mL}$ conical tube, purified and concentrated using the DNA clean \& concentrator-100 kit (Zymo Research, Cat\#: D4030) and eluted in 150 ul of water. Due to the high volume after addition of DNA binding buffer, samples were run three times through the same DNA Clean and Concentrator filter before elution. We further purified the materials eluted from the columns by adding $150 \mathrm{ul}$ (1:1) of AMPure beads RNAclean XP (Beckman coulter, A63987) and incubating for 5 min. The bound beads were pelleted with a magnet and washed twice for 30 seconds (sec) with enough freshly made $80 \%$ ethanol to submerge the beads. After removal of ethanol, the beads were allowed to dry completely before eluting with $32 \mathrm{ul}$ of water. To remove single stranded DNA and excess nucleotides, exonuclease I (Exol) treatment was performed on all samples by adding 4 ul Exol buffer and 4 ul Exol (New England Biolabs, M0293L). Samples were incubated at $37 \mathrm{C}$ for $30 \mathrm{~min}$, heat inactivated at $85 \mathrm{C}$ for $15 \mathrm{~min}$, and held at $4 \mathrm{C}$. cDNA was then amplified by adding 50 ul 2X Kapa HIFI PCR ReadyMix (Kapa Biosystems, KK2602), 10 ul of the 10 uM DRUG-seq $\mathrm{PCR}$ primer, and then running the following program:

\begin{tabular}{|c|c|c|}
\hline Temperature & Time & Cycles \\
\hline $96 \mathrm{C}$ & Preheat and pause & \\
\hline $96 \mathrm{C}$ & $1 \mathrm{~min}$ & 1 \\
\hline $98 \mathrm{C}$ & $20 \mathrm{sec}$ & \\
$58 \mathrm{C}$ & $4 \mathrm{~min}$ & 5 \\
$72 \mathrm{C}$ & $6 \mathrm{~min}$ & \\
\hline $98 \mathrm{C}$ & $20 \mathrm{sec}$ & \\
$60 \mathrm{C}$ & $30 \mathrm{sec}$ & 13 \\
$72 \mathrm{C}$ & $6 \mathrm{~min}$ & \\
\hline $72 \mathrm{C}$ & $10 \mathrm{~min}$ & \multirow{2}{*}{1} \\
$4 \mathrm{C}$ & Hold (overnight) & \\
\hline
\end{tabular}

cDNA samples were purified using the Agencourt RNA clean beads as described above but eluted with 11 ul of water. We ran 1 ul on a Bioanalyzer (Agilent, G2939BA) with DNA high sensitivity chip (Agilent) or Fragment analyzer (Agilent, M5310AA). We expect to see a wide range of fragment sizes, as represented in the figure below. Preamp abundance will be determined by 
cell type but for U2OS cells, we generally observe quantities of 1 to $5 \mathrm{ng} / \mathrm{ul}$. Size range from 200 to 6000 (representative below).

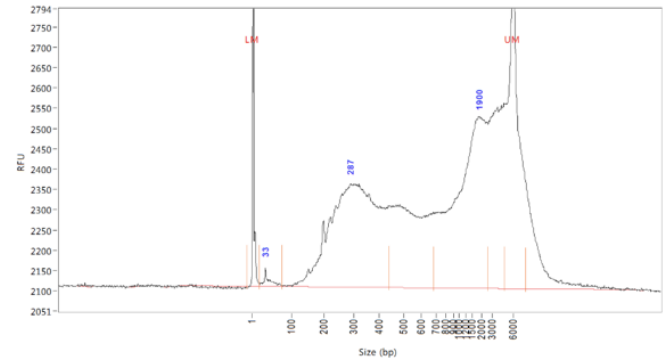

Day 4: Tagmentation, Purification and Quantification of DRUG-seq libraries

For tagmentation, 5ul of pre-amp material, measured by fragment analyzer, was mixed with nuclease-free water to a final volume of 20 ul. The 20 ul preamp was then mixed with 25 ul TD buffer and 5 ul TDE1 buffer (Nextera kit, FC-131-1096, Illumina) and incubated for 5 min at $55 \mathrm{C}$ and held at $10 \mathrm{C}$. Tagmented DNA was purified with the Qiagen MinElute PCR Purification Kit (Qiagen, Cat\#: 28004) and eluted with 25 ul nuclease-free water.

Each 25 ul sample is then PCR amplified using 15 ul NPM (Nextera XT DNA library preparation kit, FC-131-1024/FC-131-1096), 5 ul DRUG-seq_p5_PCR primer (5 uM) and 5 ul DRUG-seq indexing primer (Table S4). The PCR cycles were as follow:

\begin{tabular}{|c|c|c|}
\hline Temperature & Time & Cycles \\
\hline $72 \mathrm{C}$ & Preheat and pause & \\
\hline $72 \mathrm{C}$ & $3 \mathrm{~min}$ & 1 \\
\hline $95 \mathrm{C}$ & $30 \mathrm{sec}$ & 1 \\
\hline $95 \mathrm{C}$ & $10 \mathrm{sec}$ & 15 \\
$55 \mathrm{C}$ & $\begin{array}{l}30 \mathrm{sec} \\
30 \mathrm{sed}\end{array}$ & \\
\hline $72 \mathrm{C}$ & $\begin{array}{c}5 \mathrm{~min} \\
\text { Hold }\end{array}$ & 1 \\
\hline $4 \mathrm{C}$ & & \\
\hline
\end{tabular}

The amplicons were then purified using the Agencourt RNA clean beads as described above but this time eluted with 20 ul of nuclease-free water. The samples were then size-selected for 200-600bp fragments using a PippinHT 2\% agarose pre-cast gel cassette (Sage Science). 1 ul of the samples from the PippinHT were analyzed on a Fragment Analyzer (Agilent) using a DNF-474 High Sensitivity NGS Fragment Kit 1-6000bp (Agilent, DNF-474-0500). We generally observe approximately 5-10 ng/ul. Averaging a size of 300bp.

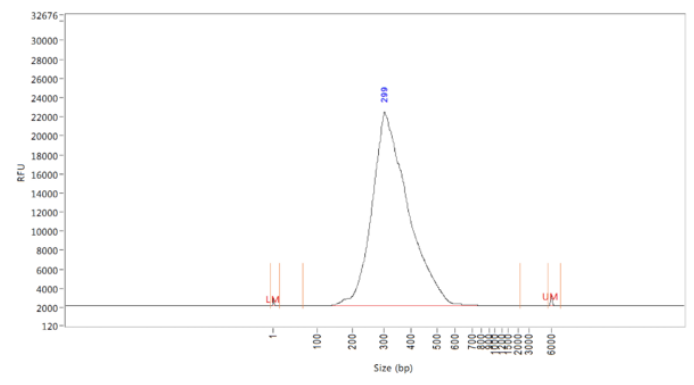

To quantify the libraries, qPCR was performed using Kapa library quantification kit for Illumina (KAPA \#KK4824 Roche \#07960140001). Following the Kapa kit manual, a pre-mix of $5 \mathrm{~mL}$ Kapa SYBR FAST qPCR Master Mix, $1 \mathrm{~mL}$ Illumina primer premix, and 200ul ROX low was 
combined and the libraries were diluted 1:20,000 with nuclease-free water. The diluted libraries as well as the 6 standards provided in each kit were plated in triplicate using 4ul per well. The reagent pre-mix was plated using $6 u$ per well. The plate was sealed and run on a QuantStudio 12k Flex with the following cycling:

\begin{tabular}{|c|c|c|}
\hline Temperature & Time & Cycles \\
\hline $95 \mathrm{C}$ & $1 \mathrm{~min}$ & 1 \\
\hline $95 \mathrm{C}$ & $15 \mathrm{sec}$ & 35 \\
$63 \mathrm{C}$ & $45 \mathrm{sec}$ & \\
\hline $95 \mathrm{C}$ & $15 \mathrm{sec}$ & \\
$60 \mathrm{C}$ & $1 \mathrm{~min} \mathrm{Hold}$ & Melt Curve Stage \\
$95 \mathrm{C}$ & $15 \mathrm{sec}$ & \\
\hline
\end{tabular}

qPCR data analysis was performed using automatic threshold and baseline settings in the QuantStudio software. The quantities calculated by the software were then dilution corrected by multiplying by the dilution factor above and size corrected following the Kapa kit manual. The libraries were normalized and pooled based on the GPCR quantities. On the following day, library denature was made and sequencing was performed on Illumina's HiSeq 4000 utilizing a custom Read 1 primer (following manufacturer's protocol).

Primer sequences (see Ye et. al., 2018 for full description)

Template switching primer AAGCAGTGGTATCAACGCAGAGTGAATrGrGrG

DRUG-seq Barcoded RT primers: AAGCAGTGGTATCAACGCAGAGTACAACAAGGTACNNNNNNNNNNTTTTTTTTTTTTT TTTTTTTTTTTTV

DRUG-seq PCR primer AAGCAGTGGTATCAACGCAGAGT

DRUG-seq_p5_PCR primer AATGATACGGCGACCACCGAGATCTACACGCCTGTCCGCGGAAGCAGTGGTATCA ACGCAGAGT*A*C

DRUG-seq indexing CAAGCAGAAGACGGCATACGAGATNNNNNNNNGTCTCGTGGGCTCGG

DRUG-seq custom read1 primer GCCTGTCCGCGGAAGCAGTGGTATCAACGCAGAGTAC

\section{Code and Data}

Raw data and processed data: GSE176150

Github: https://github.com/Novartis/DRUG-seq 